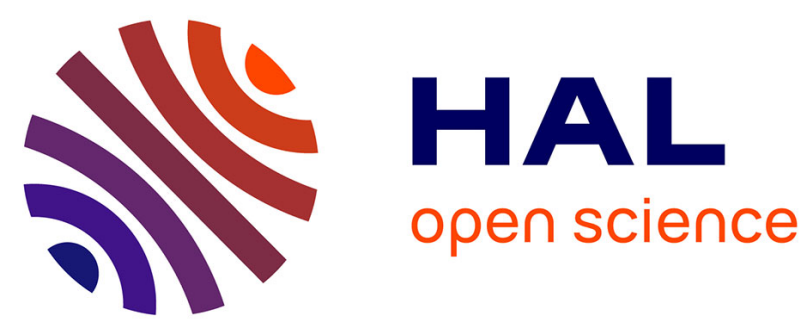

\title{
INTERACTION CHOC-SURFACE LIQUIDE : COMPARAISON CODE DE CAVITATION ET EXPÉRIMENTATION
}

\author{
N. Fargere
}

\section{- To cite this version:}

N. Fargere. INTERACTION CHOC-SURFACE LIQUIDE: COMPARAISON CODE DE CAVITATION ET EXPÉRIMENTATION. Journal de Physique IV Proceedings, 1991, 01 (C3), pp.C3-893C3-897. 10.1051/.jp4:19913125 . jpa-00249930

\section{HAL Id: jpa-00249930 https://hal.science/jpa-00249930}

Submitted on 1 Jan 1991

HAL is a multi-disciplinary open access archive for the deposit and dissemination of scientific research documents, whether they are published or not. The documents may come from teaching and research institutions in France or abroad, or from public or private research centers.
L'archive ouverte pluridisciplinaire HAL, est destinée au dépôt et à la diffusion de documents scientifiques de niveau recherche, publiés ou non, émanant des établissements d'enseignement et de recherche français ou étrangers, des laboratoires publics ou privés. 
Colloque C3, suppl. au Journal de Physique III, Vol. 1, octobre 1991

\title{
INTERACTION CHOC-SURFACE LIQUIDE : COMPARAISON CODE DE CAVITATION ET EXPERIMENTATION
}

\author{
N. FARGERE \\ Centre d'Etudes du Bouchet, F-91710 vert le Petit. France
}

\begin{abstract}
Résumé - Nous avons développé une simulation numérique qui permet de caractériser la zone de cavitation liée à l'onde de détente résultant de l'interaction d'une onde de choc sphérique subaquatique avec l'interface liquide-air. Cette simulation, prenant en compte le temps et différentes méthodes de calcul, est relativement bien corrélée par les résultats expérimentaux, obtenus sur de petites quantités d'explosif. Un passage à des quantités d'explosif plus importantes est possible par la loi de Similitude.
\end{abstract}

Abstract - Employing several methods and taking into account time in the calculations, we managed to develop a numerical code characterizing the cavitation zone due to the refraction of a subaquatic shock wave on the above surface. The results obtained with experimental means involving very small amount of explosive agree rather well with the code outputs and are likely to be extended by the mean of the Similitude law to higher weight charges.

\section{1.- Introduction}

Nous nous sommes intéressés aux mécanismes d'occurrence de la cavitation formée sous la surface, au passage d'une onde de choc sphérique subaquatique au travers de l'interface cau-air. Afin de formaliser les conditions d'apparition de ce phénomène nous nous sommes intéressés aux conjugaisons des pressions, choc incident et détente réfléchie, pression hydrostatique etc... Deux orientations différentes ont été données aux travaux. Une simulation numérique sur gros système, a partir de codes du type HEMP2, DYNA 2D ou HULL et une approche moins lourde, mais prenant peut ĉtre plus en compte l'aspect "physique", sur micro-ordinateur. Dans l'ensemble des travaux réalisés, la tension limite supportable par l'eau dans la qualité qui est la sienne lors de nos tirs est fixée à la valeur de pression de vapeur saturante. Ce choix, nous le savons, est sujet à discussion, mais il reste le plus justifiable dans notre configuration (eau industriclle non traité).

\section{2.- Modèle expérimental et expérimentation}

Pratiquement, nous travaillons avec un bassin de $0,5 \mathrm{~m}^{3}$ (section $1 \mathrm{~m}^{2}$ ) rempli d'eau. Un détonateur de $215 \mathrm{mg}$ de PETN est immergé à différentes profondeurs comprises entre $150 \mathrm{ct} 250 \mathrm{~mm}$ et activé. L'onde de choc sphérique ainsi créée se propage dans le liquide avec unc vitcsse décroissant rapidement pour devenir légèrement supersonique $(1500 \mathrm{~m} / \mathrm{s})$. L'interaction avec l'interface liquide-air se produit selon une configuration et des paramètres caractéristiques qu'il est facile de faire varier.

Plusieurs moyens d'observation et de mesure ont été utilisés. Hormis les classiques mesures de pression nous retrouvons la tomographic lascr, la micro-holographie, les films grande cadence $\left(10^{4}, 10^{6} \mathrm{im} / \mathrm{s}\right)$, la strioscopic et l'ombroscopie. Outre la zone de cavitation, que nous avons pu observer de différentes façons, nous avons aussi individualisé les modes d'apparition et de ruptures des microjets à la surface, origine du brouillard précoce accompagnant les explosions sous-marines [1].

\section{3.- Gros codes}

Les résultats obtenus avec les "gros codes" de simulation confirment les mesures de pression que nous avons réalisées dans le cadre expérimental, et nous obtenons aussi des valeurs caractéristiques (pression, vitesse...) sensiblement 
égales à celles des approches par l'application du théorème de la Similitude à notre configuration, en utilisant les parametres du modèle expérimental que nous utilisons. Malgré un maillage relativement petit, les seuils de pression trop faibles, la différence entre les densités mises en opposition trop importantes, ont été à l'origine de l'impossibilité de cerner correctement la cavitation.

D'une façon générale, ce qui peut être retiré du passage par ce type de code, c'est avant tout une vérification de la qualité des résultats expérimentaux, d'une part et des hypothèses de similitude d'autre part. Malheureusement, dans l'état actuel de leur conception, ces codes ne peuvent pas apporter des résultats exploitables pour des simulations impliquant des modélisations diphasiques, parce qu'ils nécessitent des maillages trop fins et des temps de calcul rédhibitoires.

\section{4.- Codes sur petites machines (développés in situ)}

\section{1.- Généralités}

Pratiquement, l'équation générale des pressions utilisée dans nos travaux est la suivante :

$$
\text { PHydro }+P \text { incidente }-P \text { réfléchie }=P
$$

Dans cette équation, PHydro correspond à la pression bydrostatique additionné à la pression atmosphérique. Nous faisons la supposition que toute l'onde incidente est réfléchie sur la surface et que son profil est connu. En fait, on suppose que l'on se trouve dans un milieu totalement homogène et deux sources de mêmes caractéristiques détonent au même instant, les ondes sphériques émises se rencontrant à mi-chemin et se combinant alors algébriquement.

\section{2.- Simulation selon la théorie de COLE [2]}

L'expression (1) est calculée à partir des hypothèses précédentes, en relevant tous les points où la pression est inférieure à PVs (Pression de Vapeur saturante), on considère alors qu'il y a cavitation en ce point et qu'elle durera tant que la pression locale ne dépasscra pas cette valeur. La zone de cavitation garde toujours la forme d'une calotte retournée, à concavité tournée vers la surface et atteignant le centre de détonation. Cette caractéristique n'est pas réellement admissible. Une comparaison à la silhouette expérimentale obtenue par micro-holographie n'est pas non plus encourageante.

\section{3.- Reprise de la théorie de DUBESSET et LAVERGNE [3]}

La zone de cavitation perturbe l'onde de choc incidente et la détente reffecchie. Cette dernière est particulièrement atténuée et déformée par la cavitation. En utilisant les résultats d'ARONS et YENNIE [4], DUBESSET et LAVERGNE ont tenu compte de l'érosion du front de détente. En utilisant l'équation générale de pression (1) on détermine alors le volume total sous la surface où la cavitation a eu lieu, sans préjuger de sa forme à un instant donné. La cavitation se propage vers le bas en suivant l'onde de détente. Le phénomène physique de l'érosion conduit à penser que la zone s'étend assez peu en profondeur. La forme obtenue en simulation est celle d'un calotte sphérique, plus ou moins centrée sur la charge.

La figure suivante illustre le problème posé :

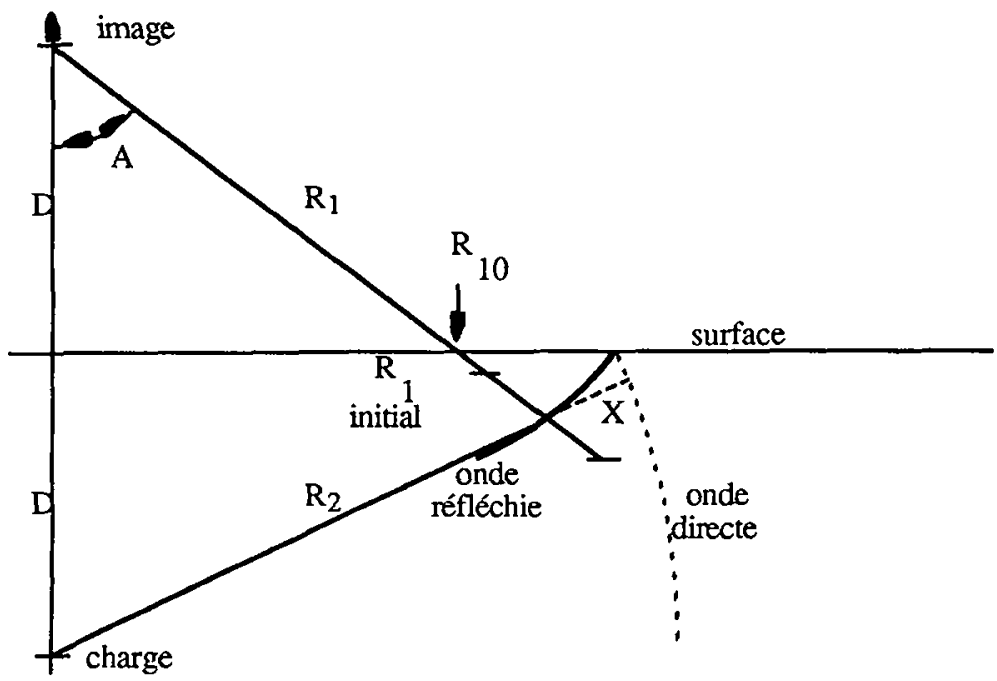

Représentation du problème en coordonnées cylindriques

L'image est l'origine des coordonnées $\left(R_{1}, A\right)$ 
En un point $\mathrm{M}$, de coordonnées cylindriques (R1, A), sur le front d'onde réfléchi, écrivons la somme des pressions :

$P h\left(R_{1}, A\right)+P\left(\frac{W^{1 / 3}}{R_{2}}, \tau\right)-P\left(\frac{W^{1 \cdot B}}{R_{1}}, 0\right)$

avec:

- la pression hydrostatique $\mathrm{Ph}\left(\mathrm{R}_{1}, \mathrm{~A}\right)$,

- la queue de pression de l'onde de choc à la distance $R_{2}$ de la charge, au temps réduit $\tau$, après l'arrivée de l'onde de choc en $M$, soit $P\left(\frac{W^{1 / 3}}{R_{2}}, \tau\right)$,

- le pic négative de pression (onde réfléchie), à la distance R1 de l'image de la charge, atténuée par la cavitation que l'on écrit alors, $-\mathrm{P}\left(\frac{\mathrm{Wi}^{1 / 3}}{\mathrm{R}_{1}}, 0\right)$.

W est la masse de la charge explosive. Wi est alors une charge image ajusté à chaque instant, pour tenir compte de l'érosion du front de détente. Wi est tel que le pic de l'onde réfléchie compense en tout point $\left(R_{1}, A\right)$, la pression hydrostatique et la pression dans la queue du front de choc, au moment où l'onde réfléchie arrive en $\left(R_{1}, A\right)$. Pour un point $M\left(R_{1}, A\right)$, situé dans la zone de cavitation, l'équation (2) doit être égale à Pvs, ainsi que nous l'avons dit en préambule.

La dynamique du calcul consiste donc à faire croître $R_{1}$, à partir de la valeur $R_{10}=D / \cos A$, correspondant au point $M$ sur la surface, pour des valeurs fixées de $A$.

Pour $R_{10}$ et $W i=W$, le premier membre de l'équation est généralement superieur à PVs, ce qui veut dire qu'il n'y a pas cavitation, et il devient égal à Pvs pour une certaine valeur de $R_{1}=R_{1}$ init (et $W i=W$ ). En effet, lorsque le rayon réfléchi pénètre dans la zone de cavitation, Wi est toujours égal à W. Lorsqu'il traverse cette zone, le pic de pression s'érode de plus en plus ce qui se traduit par une décroissance monotone de Wi. (On rappelle que les formules d'évaluation des différents paramètres sont celles de COOK[5]).

Wi passe par une valeur minimale pour la valeur de $R_{1}=R_{1}$ fin. Pour $R_{1}>R_{1}$ fin. Wi commence à croîre, la pression hydrostatique et l'onde incidente sont alors suffisantes pour compenser la pression négative, ce qui signifie que nous sommes sortis de la zone de cavitation.

La zone de cavitation pour un angle $A$ est donc comprise entre $R_{1}$ init et $R_{1}$ fin, pour lesquels, respectivement, $\mathrm{Wi}=\mathrm{W}$ et $\mathrm{Wi}=\mathrm{W} \min$.

On retrouvera les démarches des calculs dans les références citées précédemment. Nous avons ainsi cré́ le code ASTEC I (Algorithme Spatio-Temporel d'Evaluation de la Cavitation). Cependant il ne prend pas en compte le temps.

\section{4.- Evolution de ASTEC I vers ASTEC II}

La configuration expérimentale et paramétrique reste identique. Pour un angle $A$ donné on calcule $R 1$ init et $R 1$ fin. Comme dans le développement précédent à partir de R1 init nous incrémentons la distance à l'image et calculons le Wi correspondant à cette distance. L'instant où peut commencer la cavitation est celui où l'onde de choc incidente est passée et où l'onde de détente est présente. A partir de cet instant et en ce point on commence à faire varier les différents paramètres en reprenant la formule de COLE. Ces calculs sont réalisés avec la masse initiale (W) d'explosif. Ainsi la durée de cavitation est obtenue par excès. Alors, en faisant varier A dans la plage habituelle, nous sommes capables de donner une allurc de la silhouette de cavitation à des instants différents. 


\section{5.- Un accroissement dans la précision : ASTEC III}

- Recherche de la masse fictive optimale

Si l'on reprend les hypothèses de base qui nous ont conduits au code ASTEC II, nous savons que l'approximation du temps de durée de la cavitation est très fortement surévaluée en utilisant W. Il faut donc chcrcher à minimiser la masse image après le début de cavitation.

Nous savons que la fonction Wi ( $\left.\mathbf{R}_{1}\right)$ est monotone décroissante, et que ses caractéristiques sont celles de toutes les fonctions du même type, (où la masse est prise en compte). On peut donc dire que ce sera le propre de toute fonction établissant la valeur d'une masse fictive comprise entre Wi et W. Les conditions aux limites imposent que $W i\left(R_{1}\right.$ iniv) $=$ $W=W f\left(R_{1}\right.$ iniঠ $)$ et $W i\left(R_{1}\right.$ fin $)=W i \min =W f\left(R_{1}\right.$ fin $)$. En tenant compte de la monotonie, du fait que la dérivée sur le domaine doit être toujours négative croissante (ce qui conduit à ne pas annuler la dérivée seconde), la fonction qui à $R_{1}$ fait correspondre le Wf parcourant la droite passant par W et Wi min, notée Wd, est encore un majorant du Wf réel. Mais les calculs menés avec cette définition nous conduisent à un fantôme de cavitation beaucoup plus près de la réalité. Si l'on intègre maintenant dans ce raisonnement une réflexion sur les dérivées et la situation des courbes de Wi et Wf auprès des limites, il y a encore possibilité de se rapprocher de Wf Réel (Wf... est forcément fictif, puisque c'est un moyen de concrétiser l'érosion du front de détente, mais dans cette hypothèse, il y a un Wf qui est optimal quant à la durće du phénomène de cavitation au point considéré). En effet, si l'on étudie plus précisément la courbe Wi ( $\left.R_{1}\right)$, on constate que la pente cst très importante près de $R_{1}$ init et décroît en valeur absolue pour arriver à 0 , à la valeur de $R_{1}$ fin. Afin de rester au plus près de la réalité, la courbe spécifique de Wf doit être du même type.

En reprenant les considérations sur les dérivées (première et seconde) établies précédemment, on arrive à la conclusion qu'il est possible de minimiser encore Wf en le prenant égal à $(\mathrm{Wi}+\mathrm{Wd}) / 2=\mathrm{Wm}$. On précisera que les résultats obtenus pour Wd et Wm sont sensiblement équivalents quant aux fantômes, ceci étant dû à la forme particulière de la courbe Wi $\left(R_{1}\right)$.

De la même façon qu'ASTECII, en utilisant ASTEC III nous obtenons l'état dú fantôme de la zone de cavitation à un instant donné, mais d'une façon plus précise. Cette précision conceme moins la forme générale de la silhouette, que la rapidité de son évolution.

\section{5.- Discussion générale et conclusions}

Nous avons vu que les résultats obtenus en traitant le problème par de gros codes hydrodynamiques, s'ils confirment d'une manière génćrale les résultats déjà connus par la mesure ou la méthode des similitudes de COOK, sont d'un intérêt moins flagrant pour le repérage de la zone de cavitation. Il serait nécessaire de procéder à un maillage encore plus fin du système, relatif au diamètre des bulles de cavitation, ce qui paraît difficile, ne serait-ce que pour des raisons de temps calcul.

Les différentes expériences que nous connaissons qui ont été réalisées sur des sujets semblables(KAMEGAI, KLEIN, ROSENKILDE [6], L.C.MADER [7]) sont très intéressantes, mais ne se rapprochent pas beaucoup de notre modèle expérimental. Cependant, on peut tirer comme principal enscignement, que le problème soulevé est loin d'ĉtre simple, ne serait-ce que parce qu'il met en présence des milieux totalement différents cn ce qui concerne les modes de propagation des perturbations.

La première approche numérique était basée sur les hypothèses de COLE. Nous avons réalisé une simulation numérique qui donne des résultats intéressants. Cependant, après avoir pris connaissance des travaux de KEDRINSKII [8], nous avons remarqué que la zone de cavitation qu'il trouvait n'avait pas grand chose à voir avec celle que nous avions obtenue. Mais une approche telle que celle de KEDRINSKII est beaucoup trop sophistiquée pour nos moyens de calcul, et nous ne possédons pas tous les éléments, en particulier les analyses physico-chimiques de l'eau dans laquelle nous travaillons (quantité de gaz non dissout, taille des germes, etc). Le travail que nous avons conduit et dont la démarche conceptuelle ressort nettement de ces paragraphes est caractérisé par une utilisation relativement facile, et bien que donnant des résultats par excès, il permet dapprocher très correctement la zone de cavitation en fonction du temps, ainsi que le fantôme général. Des moyens d'enregistrement tels que l'holographie, l'ombroscopie nous ont permis de qualifier la modélisation /1/. Ainsi, le code que nous avons développé répond d'une façon suffiscmment précise et rapide à une question ponctuelle concernant la position et le volume de la zône de cavitation à un instant donné. Si l'on dispose de plus de temps on peut, de surcroît, caler les paramètres de la simulation de façon plus précise (principalement, réglage de la pression dite pression de cavitation). Ces réglages peuvent se faire soit au moyen d'une approche théorique quantitative (prise cn compte de la nature du liquide), soit en adoptant une démarche plus expérimentale (interprétation et prise en compte des résultats des mesures comparatives de profondeur de cavitation). 


\section{Bibliographie}

/1/ FARGERE, N, Contribution à I'Etude des effets liés à l'interaction d'une onde de choc sphérique subaquatique sur l'interface liquide-air. Thèse Université de Poitiers (1).

12/ WILLIS, R.H., Underwater Explosions. Princeton - N.J. Princeton Univ. Press (1949).

13/ DUBESSET and LAVERGNE, Calcul de cavitation dû aux explosions sous-marines à faible profondeur. Acoustica vol. 20 (1968).

14/ ARONS, AB, YENNIE DR. Energy partition in underwater explosion phenomena. Rev. Mod Phys. 20 (5/9 1948).

/5/ COOK. Underwater Explosions. Encyclopedia of Explosives Related Iterns. Vol. 10.

16/ KAMEGAI, M, KLEIN, LS, ROSENKILDE CE. Computer simulation studies on free surface reflection of underwater shock waves. US Department of Energy by the Lawrence Livermore National Laboratory contract number W. 7405 ENG 48. Presentation 16th International Symposium on schock tubes and schock waves 26-30/07. Auchen, Germany.

17/ MADER, CL. Detonations Near the Water surfacc. Los Alamos Scientific Laboratory. New Mexico 87.544 (Intemational Report) (1987).

18/ KEDRINSKII, VK. Surface Effects from an Underwater Explosion. Shumal Prikladnoï Mckkaniki i Technicheskoï Fiziki $n^{\circ} 4$ pp. 66-87 (1978). 